ITEP/TH-26/02

hep-th/0205261

\title{
Givental formula in terms of Virasoro operators
}

\author{
A. Alexandrov ${ }^{\dagger}$
}

\begin{abstract}
We present a conjecture that the universal enveloping algebra of differential operators $\frac{\partial}{\partial t_{k}}$ over $\mathbb{C}$ coincides in the origin with the universal enveloping algebra of the (Borel subalgebra of) Virasoro generators from the Kontsevich model. Thus, we can decompose any (pseudo)differential operator to a combination of the Virasoro operators. Using this decomposition we present the r.h.s. of the Givental formula [1] as a constant part of the differential operator we introduce. In the case of $\mathbb{C P}^{1}$ studied in [2], the 1.h.s. of the Givental formula is a unit, which imposes certain constraints on this differential operator. We explicitly check that these constraints are correct up to $O\left(q^{4}\right)$. We also propose a conjecture of factorization modulo Hirota equation of the differential operator introduced and check this conjecture with the same accuracy.
\end{abstract}

\section{Introduction}

It is well known that $\mathcal{N}=2$ supersymmetric sigma-model with target space $M$, defined on the genus $g$ Riemann surface, through twisting, leads to the topological field theory [3]. For $D=2$ sigma-model, the correlation functions of such topological field theory are known to be some intersection numbers on the target space, and the fields of this theory, which we call primary fields, are in one-to-one correspondence with the elements of $H^{*}(M)$ (see, for example, [4]). Thus, for any genus $g$, the free energy $F_{M}^{g}(s)$ of the theory, as a function of couplings $s$ to primary fields (the space of such couplings is called small phase space) is a generating function for such intersection numbers on the target space. The genus zero free energy $F_{M}^{0}(s)$ is described by associativity - WDVV equations [4] [5].

Coupling of the topological field theory to the topological gravity leads to the so called topological string theory. The genus $g$ free energy $\mathcal{F}_{M}^{g}(t)$ of this theory, which depends on infinitely many coupling constants $t^{1}$ (couplings to gravitational descendants), describes intersection theory on the compactified moduli spaces of genus $g$ punctured Riemann surfaces. Combining $\mathcal{F}_{M}^{g}(t)$ for all $g$ into the sum and exponentiating $\tau_{M}(t)=\exp \sum_{g=0} \hbar^{g-1} \mathcal{F}_{M}^{g}(t)$, one gets a general object which depends on the manifold and is the function of infinitely many coupling constants. We call it $\tau$-function [6]. This function can be considered from many different points of view, which lead to different conditions on $\tau_{M}(t)([4][7][8][9])$ (Virasoro constraints, dilaton and divisor equations). However, in general, they do not seem to be restrictive enough to determine $\tau_{M}(t)$ completely.

In fact, there are at least two examples of the manifold $M$ when the function $\tau_{M}$ for topological field theory with the target space $M$ turns out to be the $\tau$-function of integrable

\footnotetext{
${ }^{\dagger}$ ITEP, Moscow, Russia; e-mail: alex@gate.itep.ru

${ }^{1}$ We will denote coordinates on the big phase space as $t$, while on the small phase space, subspace of the big one, as $s$.
} 
hierarchy. The first example is the point. Its function $\tau_{\bullet}$, which corresponds to the pure topological gravity [4], is the $\tau$-function for the $\mathrm{KdV}$ hierarchy [10],[11]. It can be realized as the matrix integral (famous Kontsevich model[12])

$$
\tau_{\bullet}(T)=\frac{\int d H e^{-1 / 3 \operatorname{Tr} H^{3}+\operatorname{Tr} M^{2} H}}{\int d H e^{-\operatorname{Tr} M H^{2}}}
$$

over $N \times N$ Hermitian matrix, the coupling constants, i.e. the times from the integrable point of view, are defined as follows

$$
T_{k}=-\frac{1}{k} \operatorname{Tr} M^{-k}
$$

Invariance of this matrix integral with respect to the change of variables [13], namely,

$$
H \rightarrow H+\epsilon_{p},
$$

with $\epsilon_{p}=M^{2 p}$ leads to the Virasoro constraints $L_{n} \tau_{\bullet}\left(T_{k}\right)=0, n \geq-1$ (see, for instance, $[14],[15],[16])$ with $^{2}$

$$
L_{n}=\frac{1}{2} \sum_{k \text { odd }} k T_{k} \frac{\partial}{\partial T_{k+2 n}}+\frac{1}{4} \sum_{\substack{a+b=2 n \\ a, b \text { odd and }>0}} \frac{\partial^{2}}{\partial T_{a} \partial T_{b}}+\frac{T_{1}^{2}}{4} \delta_{n,-1}+\frac{1}{16} \delta_{n, 0}-\frac{\partial}{\partial T_{3+2 n}}, \quad n \geq-1 .
$$

These Virasoro operators form the Borel subalgebra of the Virasoro algebra,

$$
\left[L_{n}, L_{m}\right]=(n-m) L_{n+m} .
$$

The second example, $M=\mathbb{C P}^{1}$, is conjectured to correspond to the Toda hierarchy [8][17]. The $\tau$-function for it can also be represented as some matrix integral. By analogous consideration, this representation leads to the Borel subalgebra of some other Virasoro algebra which annihilates the $\tau$-function. Therefore, one could expect that the function $\tau_{M}$ associated with any manifold, is the $\tau$-function for some integrable hierarchy. This is on of the reasons why we generally call it $\tau$-function.

For several particular manifolds there exist some explicit formulas for $F_{M}^{g}$. The example of $M=\mathbb{C P}^{1}$ is, in a sense, the only foreseeable: its whole free energy vanishes on the small phase space, except genus zero and genus one, which are, correspondingly, ${ }^{3}$

$$
F_{\mathbb{C P}^{1}}^{0}=\frac{1}{2}\left(s_{P}\right)^{2} s_{Q}+e^{s_{Q}}
$$

and

$$
F_{\mathbb{C P}^{1}}^{1}=-s_{Q} / 24
$$

For $\mathbb{C P}^{2}$ the genus zero free energy is of the form

$$
F_{\mathbb{C P}^{2}}^{0}=\frac{1}{2} s_{P}\left(s_{Q}\right)^{2}+\frac{1}{2}\left(s_{P}\right)^{2} s_{R}+\sum_{d=1} N_{d}^{(0)} \frac{\left(s_{R}\right)^{3 d-1}}{(3 d-1) !} \exp \left(s_{Q} d\right),
$$

\footnotetext{
${ }^{2}$ In our paper, we use another normalization and numeration of times, that is,

$$
T_{2 m+1}=\frac{\Gamma\left(\frac{3}{2}\right)}{\Gamma\left(m+\frac{3}{2}\right)} t_{m}
$$

which comes from enumerative geometry.

${ }^{3}$ Hereafter, we denote as $s_{P}, s_{Q}$ and $s_{R}$ the three coupling constants, corresponding to the cohomology classes 1, $\omega$ and $\omega^{2}$, with $\omega$ being the 2-form $\omega \in H^{2}(M)$.
} 
and for the expansion coefficients $N_{d}^{(0)}$ there is the recursive relation [7] ${ }^{4}$

$$
N_{d}^{(0)}=(3 d-4) ! \sum_{l+k=d} \frac{N_{l}^{(0)} N_{k}^{(0)}}{(3 l-1) !(3 k-1) !} k^{2} l[3 k l+l-2 k] .
$$

For the genus one free energy

$$
F_{\mathbb{C P}^{2}}^{1}=\frac{-s_{Q}}{8}+\sum_{d=1} N_{d}^{(1)} \frac{\left(s_{R}\right)^{3 d}}{(3 d) !} \exp \left(d s_{Q}\right)
$$

analogous recursion relation was derived in [18], using Virasoro constraints for $\mathbb{C P}^{n}$ and the topological recursion relations (see also [19])

$$
N_{n}^{(1)}=N_{n}^{(0)} \frac{1}{72} n(n-1)(n-2)+\sum_{k+l=n}(3 n-1) ! \frac{N_{k}^{(0)}}{(3 k-1) !} \frac{N_{l}^{(1)}}{(3 l) !} \frac{l}{9}\left(3 k^{2}-2 k\right) .
$$

However, in general, there are no expressions for higher genera similar to (10),(12), and only a few first terms in the series like (9),(11) are found.

The problem is to construct a regular procedure for finding $F_{g}^{M}(s)$. In this paper, we make a modest contribution to solving this problem. It is based on the remarkable formula which was proposed in [1] with the help of the localization technique, and connects the Kontsevich $\tau$-function $\left(\tau_{\bullet}\right.$, function for the point on the big phase space) with the $\tau$-function for some (for instance, projective) manifold $M$ on the small phase space, with the genus zero and one contributions omitted ${ }^{5}$

$$
e^{\sum_{g>1} \hbar^{g-1} F_{M}^{g}(s)}=\left[e^{D_{M}(s)} \prod_{j} \tau_{\bullet}\left(\hbar \Delta_{j} ; t_{0}^{(j)}, t_{1}^{(j)}, \ldots\right)\right]_{t_{k}^{(i)}=T_{k}^{i}(s)} .
$$

Here $s_{i}$ are coordinates on the small phase space for the manifold $M$, indices $i, j$ run from 1 to $K=\operatorname{dim}\left(H^{*}(M)\right)$, and $D_{M}(s)$ is the bilinear pseudodifferential ${ }^{6}$ operator acting on $t_{k}^{(i)}$

$$
D_{M}(s)=\frac{\hbar}{2} \sum_{k, l=0}^{\infty} \sum_{i, j} V_{k l}^{i j}(s) \Delta_{i}^{1 / 2}(s) \Delta_{j}^{1 / 2}(s) \partial_{t_{k}^{(i)}} \partial_{t_{l}^{(j)}}
$$

$V_{k l}^{i j}(s), \Delta_{i}(s)$ and $T_{k}^{i}(s)$ being some functions which can be presented in terms of the genus zero potential $F_{M}^{0}(s)$, more precisely on its third derivatives ([1],[2]).

Formula (13) was studied in [2] for the first nontrivial example of the manifold $M, \mathbb{C P}^{1}$.

Since, as we know from the explicit form of the free energy on the small phase space for $\mathbb{C P}^{1}$ the l.h.s. of (13 is unity, formula (13) can be considered as an equation imposed on the Kontsevich $\tau$-function. We call it Song equation. ${ }^{7}$ In [2] all the necessary coefficients $\Delta, V$

\footnotetext{
${ }^{4}$ The numbers $N_{d}^{(0)}$ and $N_{d}^{(1)}$ are numbers of degree $d$ rational (elliptic) curves passing through $3 d-1$ (respectively $3 d$ ) points.

${ }^{5}$ This formula was proposed for the manifolds such that $F_{M}^{0}(s)$ determines a semi-simple Frobenius structure on the space $H^{*}(M, Q)[1]$.

${ }^{6}$ We call it pseudodifferential, since it contains infinite number of derivatives. For the sake of brevity, we sometimes call it differential operator.

${ }^{7}$ This equation is bilinear, and it can be presented in the form, reminiscent of the Hirota equation. The obvious difference is that the Hirota equation is true for any values of times, whereas the equation (13) should be considered on the one-parameter family of points, since, in this case, $V, \Delta$ and $T$ depend only on $s_{Q}$, the coupling with the 2 -form on $\mathbb{C P}^{1}$.
} 
and $T$ were calculated, and (13) was checked extensively using the expansion in $\hbar$ with the accuracy of $O\left(\hbar^{4}\right)$. This calculation involves the explicit form of $\tau_{\bullet}(t)$ as a series in times.

In our paper, we reformulate the problem of calculation of the r.h.s. in (13) for given $V$ and $T$ realizing some particular differential operator $D_{M}^{\prime}$ easily connected with $D_{M}$ (see (16)) via Virasoro operators.

Namely, the shift operator

$$
\exp \sum_{i=1}^{K} \sum_{k=2}^{\infty} T_{k}^{i}(s) \partial_{t_{k}^{(i)}}
$$

obviously commutes with $D_{M}(s)$, and for the operator

$$
D_{M}^{\prime}(s)=D_{M}(s)+\sum_{i=1}^{K} \sum_{k=2}^{\infty} T_{k}^{i}(s) \partial_{t_{k}^{(i)}}
$$

we have the equality

$$
\left[e^{D_{M}(s)} \prod_{j} \tau_{\bullet}\left(\hbar \Delta_{j} ; t_{0}^{(j)}, t_{1}^{(j)}, \ldots\right)\right]_{t_{k}^{(i)}=T_{k}^{i}(s)}=\left[e^{D_{M}^{\prime}(s)} \prod_{j} \tau_{\bullet}\left(\hbar \Delta_{j} ; t_{0}^{(j)}, t_{1}^{(j)}, \ldots\right)\right]_{t_{k}^{(i)}=0} .
$$

The general idea of the paper is to present the operator $e^{D_{M}^{\prime}}$ in the origin of the space of times $t_{k}^{(i)}$ in terms of the Virasoro operators (this mean, that we consider r.h.s. modulo term, proportional to the positive powers of the times $t)(5)$

$$
\left.e^{D_{M}^{\prime}}\right|_{t=0}=\left.\sum_{\left[l_{1}\right],\left[l_{2}\right], \ldots,\left[l_{K}\right]}^{\infty} P_{\left[l_{1}\right],\left[l_{2}\right], \ldots\left[l_{K}\right]}^{M}(s) L_{\left[l_{1}\right]} \otimes L_{\left[l_{2}\right]} \otimes \ldots \otimes L_{\left[l_{K}\right]}\right|_{t=0}
$$

Here $L_{[l]}$ are products of the Virasoro operators with the nonnegative multiplicities $m_{i}$, given by multiindices $[l]$ : for $[l]=\left(m_{-1}, m_{0}, \ldots, m_{r}\right)$ we define

$$
L_{[l]}:=L_{r}^{m_{r}} \ldots L_{0}^{m_{0}} L_{-1}^{m_{-1}}
$$

and, if all multiplicities $m_{i}$ vanish,

$$
L_{[0]}=1,
$$

where we denote $[0]:=(0,0, \ldots, 0)$. Since all the Virasoro operators of the Borel subalgebra annihilate the Kontsevich $\tau$-function, the action of the operator presented in this way on the $\tau_{\bullet}$ 's at the point $t=0$ is trivial, that is, the only nonvanishing contribution to the l.h.s. of (13) is given by the constant term proportional to (20):

$$
e^{\sum_{g>1} \hbar^{g-1} F_{M}^{g}(s)}=P_{[0],[0], \ldots[0]}^{M}(s) .
$$

In particular, the Song equation in terms of $D^{\prime}$ is

$$
\left.\exp \left[D_{\mathbb{C P}^{1}}^{\prime}\right] \tau(\hbar, x) \tau(\hbar, y)\right|_{x_{n}=y_{n}=0}=1 \text {. }
$$

In the case of $\mathbb{C P}^{1}$, one can expand the operator $D_{\mathbb{C P}^{1}}^{\prime}$ in the series in $q:=\exp \left(-\frac{s_{Q}}{2}\right)$

$$
e^{D_{\mathbb{C P} 1}^{\prime}}=1+\left.\sum_{n=1}^{\infty} q^{n} \sum_{\left[l_{1}\right],\left[l_{2}\right]} P_{\left[l_{1}\right],\left[l_{2}\right]}^{\mathbb{C P}^{1}(n)}(\hbar) L_{\left[l_{1}\right]} \otimes L_{\left[l_{2}\right]}\right|_{t=0}
$$


Since for $\mathbb{C P}^{1}$ the l.h.s. of $(13)$ is equal to 1 , the Song equation is equivalent to the condition

$$
P_{[0],[0]}^{\mathbb{C P}^{1}(n)}=0, \quad n \geq 1 .
$$

In the paper, we check this condition with the accuracy of $O\left(q^{4}\right)$.

The paper contains two conjectures. The first one which states that any differential operator in times $t$ with constant coefficients can be presented as a combination of the Virasoro operators with some multiplicities at the origin helps to deal with the Givental formula. The second conjecture, whose general structure is still unknown, in particular case of $M=\mathbb{C P}^{1}$ claims that the operator $D_{\mathbb{C P}^{1}}^{\prime}(23)$ can be factorized into the sum of two parts, which are tensor squares of differential operators, modulo Hirota equations. Both this conjectures are checked perturbatively in the example of $\mathbb{C P}^{1}$ with the accuracy of $O\left(q^{4}\right)$.

The paper is organized as follows: in section 2 we represent the main results of [2] and explicitly present the Virasoro and Hirota constraints which we use in our calculations. Sections 3 and 4 are devoted to our two conjectures, while section 5 contains some concluding remarks.

\section{Main Ingredients}

In the paper [2] it was shown that, denoting $t_{n}^{(+)}=x_{n}$ and $t_{n}^{(-)}=y_{n}$, one has the following differential operator

$$
D_{\mathbb{C P}^{1}}:=\frac{\hbar}{2} \sum_{k, l \geq 0}\left(V_{k l}^{++}\left(\partial_{x_{k}} \partial_{x_{l}}+\partial_{y_{k}} \partial_{y_{l}}\right)+2 i(-1)^{l-1} V_{k l}^{+-} \partial_{x_{k}} \partial_{y_{l}}\right),
$$

and introducing $\alpha$ being $c$-numbers

$$
\alpha_{m+1}=-\frac{(2 m+1)^{2}}{8(m+1)} \alpha_{m}, \quad \alpha_{0}=1 .
$$

we can represent times ${ }^{8} T^{+}$

$$
T_{n}^{+}=\left\{\begin{array}{cl}
0, & n=0,1, \\
-\frac{\alpha_{n-1}}{2^{n-1}} \exp \left[\frac{-(n-1) s_{Q}}{2}\right], & n \geq 2 .
\end{array}\right.
$$

In the case of $\mathbb{C P}$ we have two times on the small phase space but third derivatives of the free energy depend only on one coordinate, $s_{Q}$. This mean that all components of our construction depends on this one coordinate. $\alpha$ being $c$-numbers

$$
\alpha_{m+1}=-\frac{(2 m+1)^{2}}{8(m+1)} \alpha_{m}, \quad \alpha_{0}=1 .
$$

Coefficients $V^{++}$and $V^{+-}$in (25) can be presented as bilinear combinations of $T_{k}^{+}$similar to those in the paper ${ }^{9}[2]$ :

$$
\begin{gathered}
V_{k l}^{++}=\sum_{n=0}^{k-1} \frac{(-1)^{n}(4(l+n+1)(k-n)-1)}{(2 l+2 n+1)(2 k-2 n-1)} T_{l+n+2}^{+} T_{k-n+1}^{+}+(-1)^{k+1} \frac{T_{l+k+2}^{+}}{2 l+2 k+1}, \\
V_{k l}^{+-}=i(-1)^{l}\left[\sum_{n=0}^{k-1} \frac{2(l+2 n+1-k)}{(2 l+2 n+1)(2 k-2 n-1)} T_{l+n+2}^{+} T_{k-n+1}^{+}+2(l+k+1) \frac{T_{l+k+2}^{+}}{2 l+2 k+1}\right] .
\end{gathered}
$$

\footnotetext{
${ }^{8}$ Since $T_{n}^{-}=(-1)^{n-1} T_{n}^{+}$, we should define only one set of $T$ 's.

${ }^{9}$ The proper formula has a slight mistake therein.
} 
Taking into account that, in (27) $T_{0}^{+}=T_{1}^{+}=0$, one can explicitly substitute the Kontsevich $\tau$-function to (13) in the case $M=\mathbb{C P}^{1}$, and check the equality, expanding the operator and $\tau$-functions in the $\hbar$-series

$$
\left.\exp \left[D_{\mathbb{C P}^{1}}\right] \tau_{\bullet}(\hbar, x) \tau_{\bullet}(\hbar, y)\right|_{x_{n}=y_{n}=T_{n}^{+}}=: 1+\sum_{n=1}^{\infty} a_{n} \hbar^{n}
$$

In this way, one gets an infinite family of equations on the coefficients of the decomposition of $\tau_{\bullet}(\hbar, t)$

$$
a_{n}=0, \quad \forall n>0
$$

In the paper [2], the first three equations were explicitly checked.

The Virasoro operators corresponding to our normalization of times, are (see, for example, [20])

$$
L_{n}=\left\{\begin{array}{cc}
\sum_{m=1}^{\infty} \tilde{t}_{m} \partial_{m-1}+\frac{1}{2 \hbar} t_{0}^{2}, & n=-1, \\
\sum_{m=0}^{\infty}\left(m+\frac{1}{2}\right) \tilde{t}_{m} \partial_{m}+\frac{1}{16}, & n=0, \\
\sum_{m=0}^{\infty} \frac{\Gamma\left(n+m+\frac{3}{2}\right)}{\Gamma\left(m+\frac{1}{2}\right)} \tilde{t}_{m} \partial_{m+n}+\frac{\hbar}{2} \sum_{m=0}^{n-1}(-1)^{m+1} \frac{\Gamma\left(n-m+\frac{1}{2}\right)}{\Gamma\left(-m-\frac{1}{2}\right)} \partial_{m} \partial_{n-m-1}, & n>0,
\end{array}\right.
$$

with $\tilde{t}_{m}:=t_{m}-\delta_{m, 1}, \partial_{m}=\frac{\partial}{\partial t_{m}}$. They annihilate the Kontsevich $\tau$-function,

$$
L_{n} \tau_{\bullet}(\hbar, t)=0, n \geq-1 .
$$

It is also known that any $\mathrm{KdV} \tau$-function, in particular, the Kontsevich one, solves the Hirota bilinear equations, the first three of which being (see, for instance, [21])

$$
\begin{gathered}
\left(\hbar D_{0}^{4}-12 D_{0} D_{1}\right) \tau(t) \cdot \tau(t)=0 \\
\left(-24 D_{1}^{2}+\hbar D_{0}^{3} D_{1}+\frac{\hbar^{2}}{12} D_{0}^{6}\right) \tau(t) \cdot \tau(t)=0 \\
\left(-D_{1}^{2}+\frac{\hbar^{2}}{360} D_{0}^{6}+D_{0} D_{2}\right) \tau(t) \cdot \tau(t)=0
\end{gathered}
$$

which we denote for the sake of brevity as

$$
\mathbf{H}_{i}=0, i=1,2,3 .
$$

\section{Connection between Song and Virasoro equations}

Consider an arbitrary operator of the form

$$
B:=\partial_{1}^{n_{1}} \partial_{2}^{n_{2}} \ldots \partial_{k}^{n_{k}}, \quad n_{i} \geq 0, \quad \sum_{i=1}^{k} n_{k}>0,
$$

for some finite $k$. We experimentally check for small $k$ and $n^{10}$ that this operator can be presented as a combination of the Virasoro operators (32) at the origin of the space of times

\footnotetext{
${ }^{10}$ We expressed 27 derivatives, which are necessary for our perturbative calculation (see below), in terms of the Virasoro operators. Using explicit form of the Virasoro operators we can get some answers for actions of
} 
$t$

$$
B=\left.\sum_{m_{-1}, \ldots m_{r}=0}^{\infty} c_{m_{r}, \ldots, m_{0}, m_{-1}} L_{r}^{m_{r}} \ldots L_{0}^{m_{0}} L_{-1}^{m_{-1}}\right|_{t=0}=:\left.\sum_{[l]} c_{[l]} L_{[l]}\right|_{t=0},
$$

for some finite $r$, dependent on $n$ and $k$, where we introduced multiindex $[l]=\left(m_{-1}, m_{0}, \ldots, m_{r}\right)$. Some of the multiplicities $m_{i}$, and even all them, can vanishes.

For example,

$$
\begin{gathered}
\partial_{0}=-\left.L_{-1}\right|_{t=0}, \\
\partial_{0}^{2}=\left.L_{-1}^{2}\right|_{t=0}, \\
\partial_{0}^{3}=-\left.L_{-1}^{3}\right|_{t=0}+\frac{1}{\hbar}, \\
\partial_{2}=\left.\frac{1}{30} \hbar L_{-1}^{2}\right|_{t=0}-\left.\frac{4}{15} L_{1}\right|_{t=0} .
\end{gathered}
$$

If this is correct for arbitrary $B$, one can present any pseudodifferential operator as a combination of the Virasoro operators at the origin of the space of times $t$. Thus, one can present any tensor product of $N$ differential operators as a sum of tensor products of combinations of the Virasoro operators

$$
\left.\sum_{\left[l_{1}\right],\left[l_{2}\right], \ldots\left[l_{N}\right]}^{\infty} P_{\left[l_{1}\right],\left[l_{2}\right], \ldots,\left[l_{N}\right]} L_{\left[l_{1}\right]} \otimes L_{\left[l_{2}\right]} \otimes \ldots \otimes L_{\left[l_{N}\right]}\right|_{t=0}
$$

with $P$ being some coefficients.

This representation can be appropriate for our consideration. In particular, one has

$$
e^{D_{M}^{\prime}}=\left.\sum_{\left[l_{1}\right],\left[l_{2}\right], \ldots,\left[l_{K}\right]}^{\infty} P_{\left[l_{1}\right],\left[l_{2}\right], \ldots\left[l_{K}\right]}^{M}(s) L_{\left[l_{1}\right]} \otimes L_{\left[l_{2}\right]} \otimes \ldots \otimes L_{\left[l_{K}\right]}\right|_{t=0} .
$$

Here the operator

$$
D_{M}^{\prime}(s)=D_{M}(s)+\sum_{i=1}^{K} \sum_{k=2}^{\infty} T_{k}^{i}(s) \partial_{t_{k}^{(i)}}
$$

is the combination of the $D_{M}$ and the operator, which shifts the times $t_{k}^{(i)}=T_{k}^{i}$ to zero.

Since all the Virasoro operators of the Borel subalgebra annihilate $\tau_{\bullet}$, calculation of the action of the operator on the product of $\tau_{\bullet}$ 's in the origin is equivalent to calculating $P_{[0],[0], \ldots,[0]}$. One observes that the l.h.s. of the (13) is equal to the identity component of the decomposition of operator $e^{D_{M}^{\prime}}$ in termes of Virasoro operators

$$
e^{\sum_{g>1} \hbar^{g-1} F_{M}^{g}(s)}=P_{[0],[0], \ldots[0]}^{M}(s) .
$$

In our particular case, moving our times to the origin is equivalent to changing the operator $D$ : for the operator

$$
D_{\mathbb{C P}^{1}}^{\prime}=D_{\mathbb{C P}^{1}}+\sum_{n=2}^{\infty} T_{n}^{+}\left(\frac{\partial}{\partial x_{n}}+\frac{\partial}{\partial y_{n}}\right),
$$

the differetial operators on the tau-function, not representing them as a combination of Virasoro operators, for example

$$
\left.{\frac{\partial}{\partial T_{1}}}^{n_{0}}{\frac{\partial}{\partial T_{3}}}^{n_{1}} \tau_{\bullet}\left(T_{1}, T_{3}, \ldots\right)\right|_{T_{1}=T_{3}=\ldots=0}=\frac{1}{3}\left(\frac{3}{2}\right)^{n_{1}}\left(\frac{1}{6 \hbar}\right)^{\frac{n_{0}}{3}} \frac{\Gamma\left(-\frac{n_{0}}{3}\right)}{\Gamma\left(-n_{0}\right)} \frac{\Gamma\left(n_{1}+\frac{1}{3} n_{0}+\frac{1}{24}\right)}{\Gamma\left(\frac{1}{3} n_{0}+\frac{1}{24}\right)}
$$


the Song equation is

$$
\left.\exp \left[D^{\prime}\right] \tau_{\bullet}(\hbar, x) \tau_{\bullet}(\hbar, y)\right|_{x=y=0}=1 \text {. }
$$

Now we can check the Song equation perturbatively, not specifying the explicit form of $\tau_{\bullet}$. This is the advantage of our approach, since the approach of the paper [2] required the explicit form of $\tau_{\bullet}$. To study this equation perturbatively, we can expand the operator in the series in $q$,

$$
\exp \left[D_{\mathbb{C P}^{1}}^{\prime}\right]=: 1+\left.\sum_{n=1}^{\infty} q^{n} \sum_{\left[l_{1}\right],\left[l_{2}\right]} P_{\left[l_{1}\right],\left[l_{2}\right]}^{\mathbb{C P}^{1}(n)}(\hbar) L_{\left[l_{1}\right]} \otimes L_{\left[l_{2}\right]}\right|_{t=0},
$$

and all we must check is that

$$
P_{[0][0]}^{\mathbb{C P}^{1}(m)}=0 \quad \forall m>0
$$

We check this up to $n=3$.

The explicit expression for the operator $\left.e^{D_{\mathbb{C P} 1}^{\prime}}\right|_{x=y=0}$ acting on $\tau_{\bullet}$ 's looks like ${ }^{11}$ :

$$
\begin{aligned}
& \left.e^{D_{\mathrm{CP} 1}^{\prime}} \tau \otimes \tau\right|_{x=y=0}= \\
& =1+\left(\left[-\frac{1}{30} L_{1} \tau+\left(-\frac{7}{120} L_{-1}^{2} \tau+\frac{1}{8}\left(L_{-1} \tau\right)^{2}\right) \hbar\right] q\right. \\
& +\left[\left(\frac{1}{3600}\left(L_{1} \tau\right)^{2}+\frac{1}{3600} L_{1}^{2} \tau+\frac{1}{504} L_{2} \tau\right)+\left(\frac{109}{50400} L_{-1} \tau+\frac{53}{8400} L_{-1} L_{0} \tau\right.\right. \\
& \left.-\frac{1}{240} L_{-1} \tau L_{-1} L_{1} \tau+\frac{7}{7200} L_{-1}^{2} L_{1} \tau-\frac{7}{240} L_{-1} \tau L_{0} \tau+\frac{7}{7200} L_{-1}^{2} \tau L_{1} \tau\right) \hbar \\
& \left.+\left(\frac{49}{57600} L_{-1}^{4} \tau-\frac{7}{960} L_{-1} \tau L_{-1}^{3} \tau+\frac{499}{57600}\left(L_{-1}^{2} \tau\right)^{2}\right) \hbar^{2}\right] q^{2}+\left[\left(-\frac{97}{453600} L_{3} \tau\right.\right. \\
& \left.-\frac{1}{648000} L_{1}^{3} \tau-\frac{1}{30240} L_{1} L_{2} \tau-\frac{1}{216000} L_{1}^{2} \tau L_{1} \tau-\frac{1}{30240} L_{2} \tau L_{1} \tau\right) \\
& +\left(\frac{31}{7200}\left(L_{0} \tau\right)^{2}+\frac{14951}{3024000} L_{-1} \tau L_{1} \tau-\frac{53}{504000} L_{-1} L_{0} \tau L_{1} \tau-\frac{53}{504000} L_{-1} L_{0} L_{1} \tau\right. \\
& +\frac{1}{4032} L_{-1} \tau L_{-1} L_{2} \tau-\frac{7}{864000} L_{1}^{2} \tau L_{-1}^{2} \tau+\frac{1}{28800} L_{-1} \tau L_{-1} L_{1}^{2} \tau+\frac{1}{28800}\left(L_{-1} L_{1} \tau\right)^{2} \\
& -\frac{1}{17280} L_{-1}^{2} L_{2} \tau-\frac{1}{17280} L_{2} \tau L_{-1}^{2} \tau-\frac{61}{252000} L_{0}^{2} \tau-\frac{1319}{1814400} L_{-1} L_{1} \tau \\
& +\frac{7}{14400} L_{-1} \tau L_{0} L_{1} \tau-\frac{7}{864000} L_{-1}^{2} L_{1}^{2} \tau-\frac{151}{324000} L_{0} \tau-\frac{7}{432000} L_{-1}^{2} L_{1} \tau L_{1} \tau \\
& \left.+\frac{7}{14400} L_{-1} L_{1} \tau L_{0} \tau\right) \hbar+\left(\frac{7}{57600} L_{-1} L_{1} \tau L_{-1}^{3} \tau+\frac{1121}{1728000} L_{-1} \tau L_{-1}^{2} \tau\right. \\
& -\frac{53}{288000} L_{-1}^{3} L_{0} \tau+\frac{49}{57600} L_{-1}^{3} \tau L_{0} \tau-\frac{4567}{12096000} L_{-1}^{3} \tau-\frac{1103}{288000} L_{-1} L_{0} \tau L_{-1}^{2} \tau \\
& +\frac{7}{57600} L_{-1} \tau L_{-1}^{3} L_{1} \tau+\frac{661}{403200} L_{-1} \tau L_{-1}^{2} L_{0} \tau-\frac{499}{1728000} L_{-1}^{2} L_{1} \tau L_{-1}^{2} \tau \\
& \left.-\frac{49}{3456000} L_{-1}^{4} L_{1} \tau-\frac{49}{3456000} L_{-1}^{4} \tau L_{1} \tau\right) \hbar^{2}+\left(-\frac{6643}{13824000} L_{-1}^{4} \tau L_{-1}^{2} \tau\right. \\
& \left.\left.\left.+\frac{199}{460800}\left(L_{-1}^{3} \tau\right)^{2}+\frac{49}{460800} L_{-1} \tau L_{-1}^{5} \tau-\frac{343}{41472000} L_{-1}^{6} \tau\right) \hbar^{3}\right] q^{3}\right)\left.\right|_{t=0}+O\left(q^{4}\right) . \\
& +{ }_{-1} \tau
\end{aligned}
$$

\footnotetext{
${ }^{11}$ This form seems to be convenient, since the operator $D_{\mathbb{C P}^{1}}^{\prime}$ is symmetric in $x$ and $y$
} 
Now, if one uses the explicit expression for the $\tau$-function $\tau_{\bullet}$, conditions (47) for $m=3 n$ reproduce the conditions $a_{n}=0$ from (31). They are only those conditions that restrict the coefficients $V^{++}, V^{+-}$and $T^{+}$.

\section{Linear equation from the bilinear one}

The Song equation is quadratic in $\tau_{\bullet}$. Thus, if one manage to presents the operator $e^{D_{\mathbb{C P}^{1}}^{\prime}}$ as

$$
e^{D_{\mathbb{C P} 1}^{\prime}}=f(\hbar, q, L)^{\otimes 2}+2 \hbar q g(\hbar, q, L)^{\otimes 2},
$$

the Song equation is equivalent to the system of two linear equations

$$
\begin{aligned}
& \left.f(\hbar, q, L) \tau_{\bullet}\right|_{t=0}=1, \\
& \left.g(\hbar, q, L) \tau_{\bullet}\right|_{t=0}=0 .
\end{aligned}
$$

Here $f$ and $g$ are some operators expressed in terms of the Virasoro operators at the origin.

It turns out that the conjecture (49) is slightly incorrect, instead, with the accuracy of $O\left(q^{4}\right)$, one gets

$$
\begin{gathered}
{\left.\left[e^{D_{\mathbb{P} 1}^{\prime}}-f(\hbar, q, L)^{\otimes 2}-2 \hbar q g(\hbar, q, L)^{\otimes 2}\right] \tau \otimes \tau\right|_{t=0}=} \\
{\left.\left[\frac{\hbar}{768} q^{2} \mathbf{H}_{1}-\left(\frac{11}{12288} \mathbf{H}_{2}+\frac{21}{1024} \mathbf{H}_{3}+\frac{17}{737280} \hbar^{2} L_{-1}^{2} \mathbf{H}_{1}-\frac{1}{46080} \hbar L_{1} \mathbf{H}_{1}\right) q^{3}\right]\right|_{t=0}}
\end{gathered}
$$

where $\mathbf{H}$ 's are combinations bilinear in $\tau$ which correspond to the Hirota equations

$$
\begin{aligned}
\mathbf{H}_{1}(t=0)= & \left(\left(2 L_{-1}^{4} \tau-8 L_{-1} \tau L_{-1}^{3} \tau+6\left(L_{-1}^{2} \tau\right)^{2}\right) \hbar\right. \\
& \left.+8 L_{-1} \tau-16 L_{-1} L_{0} \tau+16 L_{-1} \tau L_{0} \tau\right)\left.\right|_{t=0} \\
\mathbf{H}_{2}(t=0)= & \left(32 L_{0} \tau-\frac{64}{3} L_{0}^{2} \tau+\frac{64}{3}\left(L_{0} \tau\right)^{2}+\left(-2 L_{-1}^{3} \tau+\frac{4}{3} L_{-1}^{3} L_{0} \tau\right.\right. \\
& \left.+2 L_{-1} \tau L_{-1}^{2} \tau-4 L_{-1} \tau L_{-1}^{2} L_{0} \tau+4 L_{-1} L_{0} \tau L_{-1}^{2} \tau-\frac{4}{3} L_{-1}^{3} \tau L_{0} \tau\right) \hbar \\
& \left.+\left(-L_{-1} \tau L_{-1}^{5} \tau+\frac{1}{6} L_{-1}^{6} \tau+\frac{5}{2} L_{-1}^{4} \tau L_{-1}^{2} \tau-\frac{5}{3}\left(L_{-1}^{3} \tau\right)^{2}\right) \hbar^{2}\right)\left.\right|_{t=0} \\
\mathbf{H}_{3}(t=0)= & \left(\left(\frac{16}{15} L_{0} \tau+\frac{8}{15} L_{-1} L_{1} \tau-\frac{8}{15} L_{-1} \tau L_{1} \tau-\frac{8}{9} L_{0}^{2} \tau+\frac{8}{9}\left(L_{0} \tau\right)^{2}\right)\right. \\
& +\left(-\frac{1}{15} L_{-1}^{3} \tau+\frac{1}{15} L_{-1} \tau L_{-1}^{2} \tau\right) \hbar+\left(\frac{1}{180} L_{-1}^{6} \tau-\frac{1}{30} L_{-1} \tau L_{-1}^{5} \tau\right. \\
& \left.\left.+\frac{1}{12} L_{-1}^{4} \tau L_{-1}^{2} \tau-\frac{1}{18}\left(L_{-1}^{3} \tau\right)^{2}\right) \hbar^{2}\right)\left.\right|_{t=0} .
\end{aligned}
$$


The operators $f$ and $g$ are, respectively,

$$
\begin{aligned}
& f(\hbar, q, L)=1+\left(-\frac{1}{60} L_{1}-\frac{7}{240} \hbar L_{-1}^{2}\right) q+\left(-\frac{13}{3150} \hbar L_{-1}+\frac{19}{1400} \hbar L_{-1} L_{0}\right. \\
& \left.+\frac{1}{1008} L_{2}+\frac{7}{14400} \hbar L_{-1}^{2} L_{1}+\frac{1}{7200} L_{1}^{2}-\frac{101}{57600} \hbar^{2} L_{-1}^{4}\right) q^{2}+\frac{1}{2}\left(\left(-\frac{97}{453600} L_{3}\right.\right. \\
& \left.-\frac{1}{648000} L_{1}^{3}-\frac{1}{30240} L_{1} L_{2}\right)+\left(-\frac{19}{42000} L_{-1} L_{0} L_{1}-\frac{59}{63000} L_{0}^{2}-\frac{7}{864000} L_{-1}^{2} L_{1}^{2}\right. \\
& \left.-\frac{1}{17280} L_{-1}^{2} L_{2}+\frac{937}{162000} L_{0}-\frac{311}{28350} L_{-1} L_{1}\right) \hbar+\left(\frac{101}{3456000} L_{-1}^{4} L_{1}\right. \\
& \left.\left.-\frac{187}{756000} L_{-1}^{3}+\frac{31}{24000} L_{-1}^{3} L_{0}\right) \hbar^{2}-\frac{793}{41472000} L_{-1}^{6} \hbar^{3}\right) q^{3}+O\left(q^{4}\right) \\
& g(\hbar, q, L)=\frac{1}{4} L_{-1}+\left(-\frac{1}{240} L_{-1} L_{1}+\frac{1}{320} \hbar L_{-1}^{3}-\frac{1}{20} L_{0}\right) q+\left(\left(\frac{97}{6300} L_{1}\right.\right. \\
& \left.+\frac{1}{1200} L_{0} L_{1}+\frac{1}{4032} L_{-1} L_{2}+\frac{1}{28800} L_{-1} L_{1}^{2}\right)+\left(-\frac{1}{2400} L_{-1}^{2}-\frac{47}{33600} L_{-1}^{2} L_{0}\right. \\
& \left.\left.-\frac{1}{19200} L_{-1}^{3} L_{1}\right) \hbar-\frac{1}{460800} L_{-1}^{5} \hbar^{2}\right) q^{2}+O\left(q^{3}\right) .
\end{aligned}
$$

\section{Conclusion}

In the paper, we presented two conjectures, which could help in the "experimental" work with the Givental formula (13), and perturbatively checked them for the simplest example of the manifold $\mathbb{C P}^{1}$. In spite of the lack of the complete prove for these conjectures, and even clear formulation of the second one, they could be of some use. One way to use them is to present the differential operator corresponding to the manifold $M$, completely in terms of the Virasoro operators with the constant coefficients $P_{\left[l_{1}\right],\left[l_{2}\right], \ldots,\left[l_{N}\right]}^{M}$ (We should mention, that this is not equivalent to present $D_{M}^{\prime}(s)$ in such form),

$$
e^{D_{M}^{\prime}(s)}=\left.\left[\exp \sum_{\left[l_{1}\right],\left[l_{2}\right], \ldots\left[l_{N}\right]}^{\infty} P_{\left[l_{1}\right],\left[l_{2}\right], \ldots,\left[l_{N}\right]}^{M} L_{\left[l_{1}\right]} \otimes L_{\left[l_{2}\right]} \otimes \ldots \otimes L_{\left[l_{N}\right]}\right]\right|_{t=0} .
$$

This is equivalent to finding the $\tau$-function of the manifold $M$ on the small phase space, if one additionally knows the genus 1 free energy (the genus zero free energy explicitly enters the coefficients in the r.h.s.). We do not know how to do this nonperturbatively, even for $M=\mathbb{C P}^{1}$. The other way is to proceed with perturbative calculations of free energies, for example, in the case of $M=\mathbb{C P}^{2}$, using formula (13) and ideas of this paper.

Another interesting way to go is to study the formula analogous to (13) such that its l.h.s. contains the free energy (with genus 0 and 1 contributions subtracted) on the big phase space [1]. It gives almost $\tau_{M}$, modulo genus zero and genus one contributions. However, the structure of the r.h.s. is far less transparent in this case.

One also can approach to the Givental formula (13) from another side. Namely, one can take some known $\tau$-function, insert it into the l.h.s. and study the structure of the differential operator at the r.h.s. The interesting example to deal with is provided by the $\tau$-function for the Generalized Kontsevich Model [?].

\section{Acknowledgments}

The author is grateful to A. Mironov for careful reading the manuscript and useful discussions, to A. Chervov, and V. Poberezhny for discussions and especially to A. Morozov 
for initiating this work and careful support during the work. I also acknowledge for the kind hospitality Les Houches summer school and ICTP spring school on superstrings and related matters. This work was partly supported by the $R F B R$ grant 00-02-16477, grant for support of young scientists 02-02-06504, Russian President's grant 00-15-99296 and grant INT AS-00-334.

\section{References}

[1] A. Givental arXiv:math.AG/0008067

[2] J. S. Song and Y. S. Song, arXiv:hep-th/0103254.

[3] E. Witten, Commun. Math. Phys. 118, 411 (1988).

[4] E. Witten, Nucl. Phys. B 340, 281 (1990).,

[5] R. Dijkgraaf, H. Verlinde and E. Verlinde, Nucl. Phys. B 352, 59 (1991). R. Dijkgraaf and E. Witten, Nucl. Phys. B 342, 486 (1990).

[6] A. Morozov, arXiv:hep-th/9502091.

[7] M. Kontsevich and Y. Manin, Commun. Math. Phys. 164 (1994) 525 [arXiv:hepth/9402147].

[8] T. Eguchi and S. K. Yang, Mod. Phys. Lett. A 9, 2893 (1994) [arXiv:hep-th/9407134]., K. Hori, Nucl. Phys. B 439, 395 (1995) [arXiv:hep-th/9411135]. T. Eguchi, Nucl. Phys. Proc. Suppl. 45BC, 149 (1996).

[9] T. Eguchi, K. Hori and C. S. Xiong, Int. J. Mod. Phys. A 12, 1743 (1997) [arXiv:hepth/9605225].

[10] M. Fukuma, H. Kawai and R. Nakayama, Commun. Math. Phys. 143, 371 (1992). M. Fukuma, H. Kawai and R. Nakayama, Commun. Math. Phys. 148, 101 (1992).

[11] M. R. Douglas, Phys. Lett. B 238, 176 (1990).

[12] M. Kontsevich, Commun. Math. Phys. 147 (1992) 1.

[13] A. Marshakov, A. Mironov and A. Morozov, ITEP-91-96. A. Mironov and A. Morozov, Phys. Lett. B 252, 47 (1990). J. Ambjorn, J. Jurkiewicz and Y. M. Makeenko, Phys. Lett. B 251, 517 (1990). H. Itoyama and Y. Matsuo, Phys. Lett. B 255, 202 (1991).

[14] E. Witten, IASSNS-HEP-91-24 In *New York 1991, Proceedings, Differential geometric methods in theoretical physics, vol. $1 * 176-216$. and Inst. Adv. Stud. Princeton - IASSNSHEP-91-24 (91/06,rec.Aug.) 43 p. and (112142).

[15] A. Marshakov, A. Mironov and A. Morozov, Phys. Lett. B 274, 280 (1992) [arXiv:hepth/9201011].

[16] S. Kharchev, A. Marshakov, A. Mironov, A. Morozov and A. Zabrodin, Nucl. Phys. B 380, 181 (1992) [arXiv:hep-th/9201013].

[17] E. Getzler, arXiv:alg.geom/0108108.

[18] T. Eguchi, M. Jinzenji and C. S. Xiong, Nucl. Phys. B 510, 608 (1998) [arXiv:hepth/9709152].

[19] E. Getzler, arXiv:alg.geom/9612004.

[20] E. Getzler, arXiv:math.ag/9805114.

[21] E. Date, M. Jimbo, M. Kashiwara and T. Miwa, RIMS-394 In *Stone, M. (ed.): Bosonization* 427-507. 